Bundesgesundheitsbl 2020 63:839-845 https://doi.org/10.1007/s00103-020-03166-z Online publiziert: 15 . Juni 2020

(c) Der/die Autor(en) 2020

\author{
Kathrin Krohn' • Michaela Pfeifer' · Petra Manzey' · Sibylle Koletzko² \\ ${ }^{1}$ Abteilung Pädiatrische Gastroenterologie und Hepatologie, Integriertes Sozialpädiatrisches Zentrum \\ (iSPZ) im Dr. von Haunerschen Kinderspital, Klinikum der Ludwig-Maximilians Universität München, \\ München, Deutschland \\ ${ }^{2}$ Abteilung Pädiatrische Gastroenterologie und Hepatologie, Kinderklinik und Kinderpoliklinik im Dr. von \\ Haunerschen Kinderspital, Klinikum der Ludwig-Maximilians-Universität München, München, \\ Deutschland
}

\title{
Chronisch entzündliche Darmerkrankungen - die biopsychosoziale Realität im Kindes- und Jugendalter
}

\section{Einleitung}

Chronisch entzündliche Darmerkrankungen (CED) umfassen den Morbus Crohn (MC), die Colitis ulcerosa (CU) und die unklassifizierte Colitis (CED$\mathrm{u}$. Die weltweite Inzidenz dieser mit hoher Morbidität einhergehenden komplexen Erkrankungen steigt an. Die aus Krankenkassendaten in Deutschland erhobene Inzidenz und Prävalenz bei Kindern und Jugendlichen ist eine der höchsten in der westlichen Welt [1]. Die Pathogenese ist multifaktoriell. Eine gestörte Interaktion zwischen mukosalem Immunsystem, Mikrobiom und umweltbedingten Risikofaktoren bei genetischer Prädisposition wird als ursächlich angenommen [2, 3].

Die Erkrankungen sind therapierbar, allerdings mit dem Risiko von Einschränkung der Lebensqualität, psychischer Komorbidität und Behinderung behaftet.

Während der Erkrankungsgipfel im Jugend- bzw. jungen Erwachsenenalter liegt, erkranken zunehmend auch Kinder unter 10 Jahren. Die Erkrankung nimmt typischerweise einen chronisch schubweisen Verlauf. Neben intestinalen Manifestationen treten bei einer Subgruppe erschwerend extraintestinale Manifestationen auf.

Bei der überwiegenden Mehrzahl der Patienten ist eine dauerhafte immunmodulierende oder immunsuppressive
Therapie notwendig, um eine Remission $\mathrm{zu}$ erzielen und zu erhalten. Zusätzlich kommen ernährungstherapeutische Interventionen und chirurgische Verfahren zum Einsatz.

Die Erkrankungen gehen mit starken Belastungen der Patienten einher einerseits durch die Erkrankung selbst und ihre Symptome, andererseits durch diagnostische Maßnahmen und potenzielle Nebenwirkungen der Therapie. Neben den medizinischen/biologischen Komplikationen treten häufig psychische und soziale Belastungen auf, die durch die Schwere der Erkrankung beeinflusst werden und umgekehrt den Erkrankungsverlauf beeinflussen können.

Die Phasen der kindlichen/jugendlichen Entwicklung gehen mit unterschiedlichen biologischen, psychischen und sozialen Risiken einher. Bei Erkrankungsbeginn in der Pubertät sind die Risiken für psychische Probleme höher als bei Erkrankungsbeginn im Kindesalter [4]. Die Krankheitsakzeptanz bei Jugendlichen und damit verbunden eine Therapiezuverlässigkeit sind dabei besondere Herausforderungen.

Diese Übersichtsarbeit dient der Darstellung der psychosozialen Folgen und Risiken bei chronisch entzündlichen Darmerkrankungen im Kindes- und Jugendalter vor dem Hintergrund der wesentlichen medizinischen Aspekte.

\section{Medizinische Aspekte der chronisch entzündlichen Darmerkrankungen}

\section{Symptome}

Bauchschmerzen, schmerzhafter Stuhldrang (Tenesmen) und (blutige) Durchfälle sind typische Symptome einer Colitis ulcerosa. Die Symptome bei Morbus Crohn sind häufig unspezifischer und umfassen Bauchschmerzen, Leistungsknick, Wachstumsstörungen und verzögerte Pubertätsentwicklung sowie Gewichtsverlust und Eisenmangelanämie mit verminderter Belastbarkeit und Müdigkeit. Die Konzentrationsfähigkeit kann beeinträchtigt sein. Kontinenzprobleme und Drangsymptomatik können insbesondere bei häufigem Durchfall auftreten. Fistulierende Verläufe bei Morbus Crohn sind je nach Lokalisation mit Schmerzen und Ausfluss verbunden $[5,6]$.

\section{Diagnostik}

Zur Sicherung der Diagnose und unter Umständen Therapieüberwachung sind Ösophagogastroduodenoskopie, Ileokoloskopie und die Dünndarmdarstellung die tragenden Säulen. Die Diagnostik sollte gemäß den internationalen sog. Portokriterien erfolgen [7]. 
Diagnostische Verfahren und die damit in der Regel verbundenen stationären Aufenthalte in der Kindergastroenterologie sind für die Patienten häufig sehr belastend. Je jünger die Kinder sind, desto schwieriger sind die Vorbereitungen für Endoskopien, insbesondere für Koloskopien. Die Darmreinigung erfolgt bei jüngeren Kindern häufig über eine Magensonde. Das Legen der Magensonde und die Darmspülung sind unangenehm, teilweise schmerzhaft.

Bildgebende Verfahren (MRT des Dünndarms und ggf. Beckenboden, Sonographie und gelegentlich die Videokapselendoskopie) sind integraler Bestandteil der diagnostischen Aufarbeitung. MRT-Untersuchungen sind insbesondere für jüngere Kinder eine große Herausforderung. Das Trinken von Mannitollösung zur Füllung des Dünndarms löst bei einigen Kindern starke Übelkeit und teilweise Bauchschmerzen aus. Bei perianalen Fisteln sind Narkoseuntersuchungen, z.T. mit Drainagen von Abszessen und Fadeneinlagen zwingend.

Bei extraintestinalen Manifestationen kommen erweiterte diagnostische Verfahren, wie u.a. die MagnetresonanzCholangiopankreatikographie (MRCP), MRT oder augenärztliche Untersuchungen zum Einsatz.

Untersuchungen und Gespräche am Krankenbett sind für die jungen Patienten häufig schambehaftet. Blutabnahmen und das Legen von Venenverweilkanülen sind für die Kinder schmerzhaft.

\section{Therapie}

Die Therapie der chronisch entzündlichen Darmerkrankungen zielt auf die Kontrolle von Beschwerden, der intestinalen Inflammation mit kompletter Heilung der Schleimhaut und Vermeidung erkrankungsbedingter Komplikationen ab. Weitere Ziele sind Optimierung des Körperwachstums, eine normale Pubertätsentwicklung und eine gesunde psychische Entwicklung sowie gute Lebensqualität. Therapienebenwirkungen sollten minimiert werden. Je nach Erkrankung (Morbus Crohn/Colitis ulcerosa/unklassifizierte CED) unterscheiden sich trotz vieler Gemeinsamkeiten die therapeutischen Möglichkeiten [8-10].

Bei Morbus Crohn wird in der Pädiatrie zur Remissionseinleitung vor allem bei Neudiagnose eine exklusive Ernährungstherapie (EET) durchgeführt [9]. Hierfür stehen verschiedene Trinknahrungen zur Verfügung, die über einen Zeitraum von 6 bis 8 Wochen ausschließlich verzehrt werden. Die Patienten dürfen in dieser Zeit keine normale Nahrung zu sich nehmen, erlaubt sind nebenbei nur Wasser und Kaugummi. Diese Therapie führt rasch zu einer Abnahme der entzündlichen Aktivität bis hin zur Mukosaheilung sowie zu einer Verbesserung des Ernährungszustandes [11]. Die Effekte sind allerdings leider nicht lang anhaltend [12]. Die EET hat den Vorteil, dass in der Phase der klinischen Remission noch ausstehende Impfungen durchgeführt werden können, bevor mit einer medikamentösen Erhaltungstherapie begonnen wird.

Aufgrund der massiven Einschränkungen während der Ernährungstherapie sind mittlerweile Ernährungstherapien auf Basis natürlicher Lebensmittel in Ergänzung zu einer partiellen Ernährungstherapie mit Trinknahrung in Erprobung [13]. Eine abschließende Empfehlung kann zurzeit noch nicht ausgesprochen werden.

Seltener kommen (bei Ablehnung der Ernährungstherapie) Glukokortikoide für eine begrenzte Zeit zum Einsatz. Die Therapie sollte aber nach spätestens 10 Wochen beendet werden. Steroide als Dauertherapie müssen unbedingt vermieden werden.

Zum Remissionserhalt bei Morbus Crohn sind in der Regel immunmodulierende oder immunsuppressive $\mathrm{Me}$ dikamente notwendig. Zum Einsatz kommen u.a. Azathioprin, Methotrexat und Biologika. TNF-alpha-Blocker (Infliximab, Adalimumab und Golimumab) sind im Gegensatz zu Azathioprin und Methotrexat, die einen verzögerten Wirkeintritt haben, auch zur remissionseinleitenden Therapie geeignet [9]. TNFalpha-Blocker sind für die Behandlung des Morbus Crohn und der Colitis ulcerosa teilweise ab 6 Jahren zugelassen. Neuere Biologika, wie Vedolizumab und Ustekinumab sind erst ab dem Erwach- senenalter zugelassen, werden jedoch bei Versagen der zugelassenen Medikamente als individueller Heilversuch („off label“) zunehmend auch bei pädiatrischen CED-Patienten eingesetzt. Der Zugang zu diesen neueren Biologika ist jedoch für Kinder erschwert, da die Beantragung bei den Krankenkassen zeitaufwendig ist, was zu verzögertem Therapiebeginn und damit Nachteilen bei den Kindern führt.

Bei der Therapie der akuten Colitis ulcerosa kommen bei leichter Aktivität 5-ASA-Präparate, sonst Glukokortikoide oder TNF-alpha-Blocker zur Remissionseinleitung zum Einsatz. Als remissionserhaltende Therapie werden Azathioprin und TNF-alpha-Blocker sowie (ebenfalls „off label“) Vedolizumab oder auch Ustekinumab eingesetzt [8].

Auch chirurgische Verfahren kommen zum Einsatz. Bei therapierefraktärer Colitis ulcerosa besteht die Möglichkeit der Kolektomie. Nach einer Kolektomie bei Colitis ulcerosa wird nach Ileostomaanlage in einem meist zweizeitigen Verfahren eine ileoanale Pouchanastomose durchgeführt, bei der aus Dünndarmschlingen ein Reservoir gebildet und eine Verbindung mit dem Anus geschaffen wird. Ziel ist die kontinenzerhaltende Rekonstruktion [8].

Beim Morbus Crohn kommen bei Stenosen oder Strikturen eine sparsame Resektion oder Strikturoplastiken zum Einsatz, wenn eine endoskopische Dilatation nicht möglich ist. Fisteln bei Morbus Crohn erfordern die interdisziplinäre kindergastroenterologische und chirurgische Versorgung (u. U. Einlage nichtschneidender Bändchen, Einsatz von Antibiotika und Biologika; [10]).

Einige Kinder müssen bei hochgradigen tief sitzenden Rektumstenosen oder Rektumbefall mit einem permanenten Stoma versorgt werden.

\section{Nebenwirkungen}

Die Chancen auf eine Mukosaheilung sind heutzutage durch den Einsatz der modernen Biologika höher als noch vor 2 Jahrzehnten. Therapieentscheidungen unter Berücksichtigung der Krankheitsaktivität und Risikoabwägung stellen eine Herausforderung für die behandeln- 
den Kindergastroenterologen dar. Hierbei kommt der (altersgerechten) Aufklärung der jungen Patienten und ihrer Eltern ein hoher Stellenwert zu.

Typische Nebenwirkungen und Risiken der immunsuppressiven Medikamente sind vermehrte Infektanfälligkeit, Unverträglichkeiten, Blutbildveränderungen, Pankreatitis, Hepatitis, Hautinfektionen, Bauchschmerzen sowie ein gegenüber Gesunden erhöhtes Malignomrisiko. Letzteres ist besonders für Hauttumoren und Lymphome unter Azathioprin beschrieben worden [14]. Bei der Nutzen-Risiko-Abwägung ist $\mathrm{zu}$ beachten, dass die Erkrankung per se mit einem erhöhten Malignomrisiko einhergeht. Besonders das Darmkrebsrisiko erhöht sich bei schlecht kontrollierter Entzündung oder bei gleichzeitigem Vorliegen einer primär sklerosierenden Cholangitis [15].

Von den bekannten typischen Steroidnebenwirkungen sind für die Kinder und Jugendlichen die cushingoide Facies („Vollmondgesicht“), Gewichtszunahme mit Ausbildung von Striae und Akne mitunter so belastend, dass sie nur schwer für eine evtl. später notwendige erneute Therapie zu motivieren sind.

Methotrexat löst bei einigen Patienten starke Übelkeit aus, was zum Abbruch der Therapie führen kann. Am Tag nach der einmal wöchentlichen Medikamenteneinnahme fühlen sich viele Patienten unwohl.

Während einige Nebenwirkungen vorwiegend biologischer Natur sind, haben andere Therapien soziale und psychische Auswirkungen.

Die Ernährungstherapie mit Trinknahrung wird von ärztlicher Seite gern empfohlen, da sie eine (medizinisch gesehen) besonders nebenwirkungsarme Möglichkeit der Remissionseinleitung bei Morbus Crohn mit einer Chance auf Mukosaheilung bei gleichzeitig günstiger Beeinflussung der beeinträchtigten nutritiven Situation mit Besserung von Muskelmasse und Knochenqualität bietet. Für die Kinder und Jugendlichen und ihre Familien ist die Therapie allerdings belastend, da die Patienten für 6 bis 8 Wochen von einem wichtigen Teil des sozialen Familienlebens - den gemeinsamen Mahlzeiten - ausgeschlos-

Bundesgesundheitsbl 2020 • 63:839-845 https://doi.org/10.1007/s00103-020-03166-z

(c) Der/die Autor(en) 2020

K. Krohn · M. Pfeifer · P. Manzey · S. Koletzko

\section{Chronisch entzündliche Darmerkrankungen - die biopsychosoziale Realität im Kindes- und Jugendalter}

\section{Zusammenfassung}

Chronisch entzündliche Darmerkrankungen (CED) im Kindes- und Jugendalter nehmen zu. Diese komplexen Erkrankungen haben typischerweise einen unvorhersehbaren und oft schubweisen Verlauf. Diese Übersichtsarbeit dient der Darstellung der psychosozialen Folgen und Risiken bei chronisch entzündlichen Darmerkrankungen im Kindes- und Jugendalter vor dem Hintergrund der wesentlichen medizinischen Aspekte.

Diagnostik und Therapie der CED sollten von Kindergastroenterologen gesteuert werden. Obere und untere Endoskopie mit Stufenbiopsien und bildgebende Verfahren sind die Eckpfeiler der Diagnostik. Therapeutisch kommen immunmodulierende und immunsuppressive Medikamente, Ernährungstherapie und chirurgische Interventionen zum Einsatz. Die Erkrankung selbst mit ihren vielfältigen Beschwerden und Kom- plikationen, aber auch die diagnostischen und therapeutischen Verfahren und die Sorge vor Nebenwirkungen sind für die jungen Patienten und ihre Familien mit vielfältigen Belastungen verbunden. Einschränkungen der Lebensqualität, psychische Erkrankungen und negative Auswirkungen auf Sozialleben, Schule und Berufsausbildung können die Folge sein. Eine interdisziplinäre umfassende Betreuung der Patienten unter Einbeziehung der verschiedenen Berufsgruppen (ärztlich, psychologisch, sozialpädagogisch, ernährungstherapeutisch und pflegerisch) sind notwendige Voraussetzungen, diesen komplex kranken Kindern und Jugendlichen sowie ihren Familien gerecht zu werden.

\section{Schlüsselwörter}

Morbus Crohn · Colitis ulcerosa · Psychosozial . Interdisziplinär · Lebensqualität

\section{Inflammatory bowel diseases-the biopsychosocial reality in childhood and adolescence}

\section{Abstract}

The prevalence of inflammatory bowel diseases (IBD) in children is rising. These complex diseases typically take an unpredictable course with disease flares. This review serves to illustrate the psychosocial consequences and risks of inflammatory bowel diseases in children and adolescents against the background of the essential medical aspects. Pediatric gastroenterologists should perform diagnostic procedures and medical care. Central elements of diagnostic workup include upper and lower endoscopy with multiple biopsies and imaging procedures. Therapeutic options comprise immunomodulating and immunosuppressive agents, nutrition therapy and surgical interventions. With its various symptoms, complications, diagnostic procedures, and therapies, including side effect concerns, the disease is challenging for young patients and their families. Quality of life, social life, and education may be impaired. There is also an increased risk for mental-health problems. An interdisciplinary approach with the involvement of various healthcare professions such as medical doctors, psychologists, social workers, dieticians, and nurses is necessary to satisfy the needs of children and adolescents with these complex medical conditions and their families.

\section{Keywords}

Crohn's disease - Ulcerative colitis - Psychosocial · Interdisciplinary · Quality of life sen sind. Daher sind die meisten Kinder und Jugendlichen nicht bereit, die exklusive Ernährungstherapie ein zweites Mal durchzuführen.

Während die Kolektomie bei schwerer therapieresistenter Colitis ulcerosa eine wichtige chirurgische Therapieoption darstellt, ist eine (vorrübergehende) Stomaanlage gerade für jugendliche Patienten häufig zunächst unvorstellbar. Bei entsprechendem Leidensdruck stimmen im Laufe der Zeit viele Jugendliche bei entsprechender Indikation zu und profitieren dann von einem gebesserten Allge- 
meinzustand. Die psychosozialen Folgen dürfen jedoch nicht unterschätzt werden. Ein Stoma birgt ein erhöhtes Risiko für psychische Erkrankungen [16].

\section{Partnerschaft, Sexualität und Familienplanung}

Die ebenfalls bedeutsamen Themen Partnerschaft, Sexualität, Verhütung und Familienplanung werden sowohl im Rahmen der ärztlichen Betreuung wie auch in psychologischen und sozialen Beratungsgesprächen thematisiert. Gerade bei Vorliegen von Fisteln und anderen perianalen Veränderungen bestehen bei Jugendlichen große Unsicherheiten bei ersten sexuellen Erfahrungen. Eine Beratung zu Methoden der Schwangerschaftsverhütung sollte stattfinden. Dies ist insbesondere bei Therapie mit Methotrexat aufgrund der Teratogenität von Methotrexat bedeutsam. Die Patientinnen sollten über Alternativen zur oralen Kontrazeption, die insbesondere bei Dünndarmbefall und Durchfällen unzuverlässig ist, aufgeklärt werden.

Die Jugendlichen beschäftigen sich auch mit Fragen bezüglich späterer Familiengründung und Fertilität. Mädchen müssen ggf. über ein erhöhtes Risiko von Infertilität nach Colektomie und ileoanaler Pouchanastomose aufgeklärt werden $[17,18]$.

\section{Psychologische Aspekte der chronisch entzündlichen Darmerkrankungen}

Die Diagnose einer chronisch entzündlichen Darmerkrankung ist ein einschneidendes kritisches Ereignis nicht nur für die betroffenen Kinder und Jugendlichen, sondern auch für ihre Familien. Sie sind plötzlich damit konfrontiert, ihr ganzes zukünftiges Leben mit einer unheilbaren Erkrankung zu verbringen, deren Krankheitsverlauf nicht vorhergesagt werden kann und die möglicherweise mit einer deutlichen Beeinträchtigung der Lebensqualität, ihrer beruflichen Ausrichtung und des sozialen Lebens einhergeht. Nicht nur die Erkrankung, sondern auch die Therapien bereiten vielen Patienten Sorgen. Die Sorgen der Eltern sind hierbei häufig größer als die der Kinder und
Jugendlichen. Die Patienten spüren die Belastung und die Trauer ihrer Eltern und möchten sie häufig nicht noch durch ihre eigenen Sorgen zusätzlich belasten. Ein Teil der Kinder und Jugendlichen beginnt, die Sorgen für sich zu behalten.

\section{Psychische Komorbiditäten}

Kinder und Jugendliche mit chronisch entzündlicher Darmerkrankung haben gegenüber somatisch gesunden Altersgenossen ein erhöhtes Risiko für psychische Erkrankungen [19, 20]. Verschiedene Risikofaktoren für psychische Erkrankungen bei CED sind beschrieben, wie niedriger sozioökonomischer Status, Gebrauch von Glukokortikoiden, elterlicher Stress, Beginn der Erkrankung im Jugendlichen- versus Kindesalter [21].

Kinder mit chronisch entzündlicher Darmerkrankung und chronisch aktiven Krankheitsverläufen können Opfer von Mobbing und Hänseleien werden, insbesondere bei Durchfällen, imperativem Stuhldrang, Inkontinenz oder Medikamentennebenwirkungen (z. B. cushingoide Facies oder Gewichtszunahme bei Steroidtherapie).

Die häufigsten psychischen Komorbiditäten sind Depressionen und Angststörungen. Das Risiko depressiver oder Angstsymptome korreliert mit höherer Krankheitsaktivität [21, 22]. Je kürzer der Erkrankungsbeginn zurückliegt, desto höher ist das Risiko für psychische Störungen [22, 23]. Bei zusätzlicher Depression oder Angststörung besteht ein erhöhtes Risiko für wiederholte CED-assoziierte stationäre Aufnahmen [24].

Je nach Untersuchung wird das Risiko für psychische Störungen unterschiedlich eingeschätzt [25]. Die Untersuchungsmethoden unterscheiden sich stark. Während viele Studien nur mithilfe von Fragebögen oder anhand der Dokumentation von ICD-10-Diagnosen durchgeführt wurden, gibt es nur wenige Untersuchungen, in denen zusätzlich diagnostische Interviews geführt wurden [22, 26, 27].

In der in Deutschland durchgeführten Studie von Engelmann et al. erfüllten über $50 \%$ der Kinder und Jugendlichen einer CED-Kohorte DSM-IV-Kriterien für eine oder mehrere psychiatrische Er- krankungen. Die Patienten wurden nicht nur mithilfe von Fragebögen zur Lebensqualität (HRQoL IMPACT III und QoL EQ-5D) untersucht, sondern zusätzlich wurden diagnostische Interviews mit Eltern und Patienten geführt. Die am häufigsten diagnostizierten Störungen waren Anpassungsstörungen, Depressionen und Angststörungen [26]. Nur ein Bruchteil dieser Patienten hatte bereits Kontakt zu Psychotherapeuten oder Kinder- und Jugendpsychiatern.

Die Diagnostik psychischer Erkrankungen kann durch Überlappungen der Symptomatik mit denen der CED (bspw. Bauchschmerzen, Erschöpfung, Schulfehlzeiten [28]) erschwert sein. Sie sollte daher in enger Kooperation zwischen Psychologen und Ärzten erfolgen. Auch bei Entscheidungen zur Priorisierung medizinischer Therapieintensivierung und/oder psychotherapeutischer Interventionen ist die enge Absprache der medizinischen und psychologischen Disziplinen notwendig.

\section{Therapieadhärenz und Compliance}

Ein Problem bei der Durchführung einer erfolgreichen remissionserhaltenden Therapie ist die fehlende Therapieadhärenz oder Compliance insbesondere bei jugendlichen Patienten. LeLeiko et al. untersuchten die Therapieadhärenz von pädiatrischen CED-Patienten, die mit 5-ASA und/oder 6-Mercaptopurin therapiert wurden, mithilfe eines elektronischen Monitorings der Medikamentenentnahme aus dem Behältnis [29]. Die Therapieadhärenz war umso schlechter, je älter die jugendlichen Patienten waren. Von den über 15-jährigen nahmen nur noch max. $25 \%$ der Patienten 5-ASA und max. $15 \%$ der Patienten 6-Mercaptopurin wie verordnet ein. Bei jüngeren Kindern war die Therapieadhärenz besser, vermutlich, weil die Eltern die Medikamenteneinnahme noch mehr kontrollierten. Erstaunlicherweise verbesserte eine erhöhte Krankheitsaktivität nicht die Therapieadhärenz.

Die Problematik der schlechten Compliance gerade im jugendlichen Alter, in dem sich die Heranwachsenden zunehmend von ihren Eltern lösen, ist ein Bereich, der in der Transitionsvorbereitung 
vor Übergang in die Erwachsenengastroenterologie eine große Rolle spielt. Wichtig ist der kontinuierliche Kontakt mit den Patienten, um ein Klima des Vertrauens zuschaffen, in dem Complianceprobleme besprochen werden können. Auch die Eltern benötigen Unterstützung, damit die Jugendlichen die notwendige Autonomie ihrem Alter entsprechend erreichen können.

Psychologische Unterstützung, Screening auf psychische Beeinträchtigungen und Evaluation von Compliance- und Adhärenzproblemen bei CED-Patienten werden in Leitlinien und von den Autoren o.g. Studien empfohlen $[8,20,22,26]$. In den meisten medizinischen Versorgungseinrichtungen steht allerdings kein oder wenig psychologisches Personal zur Verfügung, um Risikofaktoren gezielt zu erfragen und bei Bedarf zu beraten und ggf. eine Psychotherapie zu bahnen. Häufig bleiben daher psychische Erkrankungen von Patienten mit CED unterdiagnostiziert und unbehandelt $[20,26,30]$.

\section{Soziale Aspekte der chronisch entzündlichen Darm- erkrankungen}

Der überwiegende Anteil der Patienten mit chronisch entzündlichen Darmerkrankungen kann ohne größere Einschränkungen am sozialen und schulischen Leben teilnehmen. Bei Kindern mit chronisch aktiven Krankheitsverläufen oder häufigen Erkrankungsschüben können jedoch massive Beeinträchtigungen auftreten. Bei der Betreuung von CED-Patienten ist daher die enge Kooperation von ärztlichem Team und sozialer Beratung sinnvoll und wichtig.

\section{Schule, Ausbildung und soziales Umfeld}

Schulische Fehlzeiten, die durch die Symptome der Erkrankung, aber auch durch diagnostische oder therapeutische Interventionen bedingt sein können, summieren sich, sodass die Ausbildung dadurch leidet. Circa $15 \%$ der pädiatrischen Patienten mit CED fehlten einer deutschlandweiten Studie zufolge wiederholt und für längere Zeit in der Schule [31]. Über ein Drittel der befragten Patienten be- richteten darüber, dass ihre Bedürfnisse in der Schule oder Ausbildung nicht ausreichend berücksichtigt werden.

Durch Bauchschmerzen und erhöhte Entzündungsaktivität kann die Konzentrationsfähigkeit beeinträchtigt sein. Patienten mit Durchfall müssen während des Unterrichts oder auch während Prüfungen gelegentlich die Toilette aufsuchen. Bei morgendlichen Durchfällen kommen sie eventuell erst nach Schulbeginn zum Unterricht. Hier ist viel Aufklärungsarbeit in den Schulen und bei den Lehrern gefragt. Noch immer sind Erkrankungen, die mit Ausscheidungsstörungen einhergehen, in unserer Gesellschaft ein Tabuthema.

Die Sorge vor Durchfall und Schwierigkeiten, die nächste Toilette rechtzeitig $\mathrm{zu}$ erreichen, schränkt einige Jugendliche in ihrem Aktionsradius deutlich ein und hält sie teilweise davon ab, an schulischen oder außerschulischen Aktivitäten teilzunehmen. Hier gibt es zwar Hilfestellungen (z. B. Toilettenfinder-Apps oder EURO-Schlüssel für behindertengerechte Toiletten). Dies erlöst die jungen Patienten jedoch nicht von der Sorge, wie ihre Freunde oder Begleiter auf häufige Toilettengänge oder gar Inkontinenz reagieren.

Gespräche der Eltern mit den Lehrkräften über die spezifischen Beeinträchtigungen ihrer Kinder sind in der Regel sehr hilfreich, damit individuelle Regelungen zu Unterstützungen, Nachholarbeiten, Teilnahme am Sportunterricht etc. getroffen werden können. Ziel sollte die Ermöglichung der regelmäßigen Teilnahme am Schulunterricht (u. U. mit Einschränkungen) sein.

Zur Unterstützung der Patienten können Anträge auf Nachteilsausgleich gestellt werden. Hierdurch sollen erkrankungsbedingte Nachteile kompensiert werden. Beantragt werden können z.B. Zeitzuschläge bei Prüfungen, Genehmigung zur Toilettenbenutzung während des Unterrichts und bei Prüfungen, ggf. benotungsfreie Teilnahme am Sportunterricht. Bei extraintestinalen Manifestationen mit Arthralgien oder Arthritis kann die Benutzung von Aufzügen und die Bereitstellung eines zweiten Büchersatzes sinnvoll sein.
Die gesetzlichen Regelungen zu Nachteilsausgleichen unterscheiden sich von Bundesland zu Bundesland.

\section{Schwerbehindertenausweis}

Ein Schwerbehindertenausweis (SBA) kann für Kinder mit chronisch entzündlicher Darmerkrankung, insbesondere bei schwereren Verläufen, beantragt werden. Die Feststellung der Behinderung erfolgt nach den „versorgungsmedizinischen Grundsätzen“, die Anhaltswerte über die Höhe des Grades der Behinderung enthalten. Die versorgungsmedizinischen Grundsätze können beim Bundesministerium für Arbeit und Soziales unter www.bmas.de $>$ Service $>$ Publikationen in der Versorgungsmedizin-Verordnung eingesehen werden. Der Grad der Behinderung wird bei jedem Erkrankten vom Versorgungsamt individuell festgelegt. Für eine Einschätzung und Hilfestellung bei der Frage, ob und wie ein SBA beantragt wird, ist eine soziale Beratung unbedingt empfehlenswert, die neben den formalen Rahmenbedingungen auch darüber hinausgehende individuelle Aspekte beleuchtet („Was macht ein Schwerbehindertenausweis mit mir?", „Entstehen mir Nachteile, wenn ich einen SBA habe?“, „Stigmatisiert mich ein SBA?").

\section{Kinderkrankengeld}

Bei Kindern mit häufigen Krankheitsschüben oder chronisch aktiven Verläufen sind die Familien häufig auch mit finanziellen Sorgen belastet. Häufige Abwesenheiten vom Arbeitsplatz können bei den Eltern zu beruflichen Nachteilen führen.

Eltern gesetzlich krankenversicherter Kinder können bis zur Vollendung des 12. Lebensjahres pro Elternteil für bis zu 10 Tage pro Jahr Krankengeld bei Erkrankung des Kindes beantragen. Privatversicherte haben keinen Anspruch auf Kinderkrankengeld.

Auch wenn die gesetzlichen Rahmenbedingungen eine Erleichterung für die Eltern darstellen können, so darf nicht vergessen werden, dass gehäufte Fehlzeiten am Arbeitsplatz von Arbeitgebern und Kollegen nicht selten negativ aufge- 
nommen werden und wenig Verständnis für die Situation der Familien mit chronisch erkrankten Kindern herrscht.

Unterstützung und Beratung können die Patienten und Eltern bei Selbsthilfegruppen, bspw. der Deutschen Morbus Crohn/Colitis ulcerosa Vereinigung e. V. (www.dccv.de), erhalten.

\section{Interdisziplinäre Versorgung}

Für die Versorgung von Kindern und Jugendlichen mit chronisch entzündlichen Darmerkrankungen ist ein interdisziplinäres Team eine notwendige Voraussetzung. Neben der medizinischen „biologischen" Versorgung hat auch die psychosoziale Betreuung einen hohen Stellenwert.

Die medizinische Versorgung beinhaltet neben der spezialisierten kindergastroenterologischen Betreuung auch die kinderärztliche Betreuung vor Ort sowie eine Vielzahl weiterer ärztlicher Disziplinen (Kinderradiologen, Kinderchirurgen, ggf. Proktochirurgie, Infektiologen, Endokrinologen, Pharmakologen, Humangenetiker, internistische Gastroenterologen, Dermatologen, Augenärzte, Kinderrheumatologen u.a.). Dazu kommen eine Ernährungsfachkraft und kindergastroenterologisch spezialisierte Pflegekräfte, bei Bedarf eine Stomaberatung.

Zur psychosozialen Betreuung sind Psychologen und Sozialpädagogen mit Einbindung im kindergastroenterologischen Team für eine fundierte krankheitsspezifische Beratung und Begleitung essenziell. Die enge Zusammenarbeit gewährleistet ein niederschwelliges Beratungs- und Diagnostikangebot für die Patienten und deren Eltern vom Zeitpunkt der CED-Diagnose an, was außerhalb der klinischen Versorgung mit Hürden verbunden ist. Die Patienten haben häufig Sorge, durch das Aufsuchen von Psychologen stigmatisiert zu werden, und scheuen den Kontakt. Interdisziplinäre Versorgungsstrukturen können helfen, die Probleme zu minimieren und den Zugang zu Psychologen und sozialer Beratung zu erleichtern.

Ein Modul der ambulanten interdisziplinären Betreuung können sozialpädiatrische Zentren mit einer spezialisier- ten kindergastroenterologischen Fachabteilung sein. Eine enge Verzahnung mit den Bereichen der Akutmedizin und stationären Versorgung der CED-Patienten muss dabei gewährleistet sein.

\section{Fazit}

Die biopsychosoziale Realität für $\mathrm{Pa}$ tienten mit chronisch entzündlichen Darmerkrankungen beinhaltet ein vielfältiges Spektrum an medizinischen, psychischen und sozialen Herausforderungen. Nicht nur die Patienten, auch die Eltern und Geschwister sind Belastungen unterworfen. Eine integrierte interdisziplinäre Betreuung unter Einbeziehung der psychosozialen Berufsgruppen ist erforderlich, um die Versorgung dieser Patienten und ihrer Familien zu verbessern. Hierfür müssen vielerorts neue oder zusätzliche Strukturen geschaffen werden.

\section{Korrespondenzadresse}

\section{Dr. med. Kathrin Krohn}

Abteilung Pädiatrische Gastroenterologie und Hepatologie, Integriertes Sozialpädiatrisches Zentrum (iSPZ) im Dr. von Haunerschen Kinderspital, Klinikum der Ludwig-Maximilians Universität München

Lindwurmstr. 4, 80337 München, Deutschland Kathrin.Krohn@med.uni-muenchen.de

Funding. Open Access funding provided by Projekt DEAL.

\section{Einhaltung ethischer Richtlinien}

Interessenkonflikt. K. Krohn erhielt Vortragshonorarevon den Firmen Baxter und Takeda ohne Zusammenhang zu der vorliegenden Arbeit. S. Koletzko erhielt für wissenschaftliche Projekte finanzielle Unterstützung an das LMU Klinikum von den Firmen Mead Johnson, Nestec Nutrition und BioGaia sowie Honorare für wissenschaftliche Beratung oder Vortragstätigkeit von den Firman Nestle, Danone, Biocodex, Shire, Abbvie, RBiopharm, Vifor, Pharmacosmos, Celgene, ThermoFisher, Janssen, Takeda und Pfizer, ohne Zusammenhang zu der vorliegenden Arbeit. M. Pfeifer und P. Manzey geben an, dass kein Interessenkonflikt besteht.

Für diesen Beitrag wurden von den Autoren keine Studien an Menschen oder Tieren durchgeführt. Für die aufgeführten Studien gelten die jeweils dort angegebenen ethischen Richtlinien.

Open Access. Dieser Artikel wird unter der Creative Commons Namensnennung 4.0 International Lizenz veröffentlicht, welche die Nutzung, Vervielfältigung,
Bearbeitung, Verbreitung und Wiedergabe in jeglichem Medium und Format erlaubt, sofern Sie den/die ursprünglichen Autor(en) und die Quelle ordnungsgemäß nennen, einen Link zur Creative Commons Lizenz beifügen und angeben, ob Änderungen vorgenommen wurden.

Die in diesem Artikel enthaltenen Bilder und sonstiges Drittmaterial unterliegen ebenfalls der genannten Creative Commons Lizenz, sofern sich aus der Abbildungslegende nichts anderes ergibt. Sofern das betreffende Material nicht unter der genannten Creative Commons Lizenz steht und die betreffende Handlung nicht nach gesetzlichen Vorschriften erlaubt ist, ist für die oben aufgeführten Weiterverwendungen des $\mathrm{Ma}$ terials die Einwilligung des jeweiligen Rechteinhabers einzuholen.

Weitere Details zur Lizenz entnehmen Sie bitte der Lizenzinformation auf http://creativecommons.org/ licenses/by/4.0/deed.de.

\section{Literatur}

1. Wittig R, Albers L, Koletzko S, Saam J, von Kries R (2019) Pediatric chronic inflammatory bowel disease in a German statutory health INSURANCEincidence rates from 2009 to 2012. J Pediatr Gastroenterol Nutr 68(2):244-250

2. Schwerd T, Koletzko S (2019) Darmmikrobiom und chronisch-entzündliche Darmerkrankungen. Monatsschr Kinderheilkd 167:420-425

3. Knights D, Lassen KG, Xavier RJ (2013) Advances in inflammatory bowel disease pathogenesis: linking host genetics andthe microbiome. Gut 62(10):1505-1510

4. Szigethy E, Levy-Warren A, Whitton S, Bousvaros A, Gauvreau K, Leichtner AM, Beardslee WR (2004) Depressive symptoms and inflammatory bowel disease in children and adolescents: acrosssectional study. J Pediatr Gastroenterol Nutr 39(4):395-403

5. Bequet $E$, Sarter H, Fumery $M$ et al (2017) Incidence and phenotype at diagnosis of veryearly-onset compared with later-onset paediatric inflammatory bowel disease: a population-based study [1988-2011.J Crohns Colitis 11(5):519-526

6. Buderus S, Scholz D, Behrens R et al (2015) Inflammatory bowel disease in pediatric patients: characteristics of newly diagnosed patients from the CEDATA-GPGE registry. Dtsch Arztebl Int 112(8):121-127

7. Levine A, KoletzkoS, Turner Detal (2014) ESPGHAN revised porto criteria for the diagnosis of inflammatory bowel disease inchildren and adolescents. J Pediatr Gastroenterol Nutr 58(6):795-806

8. Turner D, Ruemmele FM, Orlanski-Meyer E (2018) Management of paediatric ulcerative colitis, part 1: ambulatory care-an evidence-based guideline from European Crohn's and colitis organization and European society of paediatric gastroenterology, hepatology and nutrition. J Pediatr Gastroenterol Nutr 67(2):257-291

9. Ruemmele FM, Veres G, Kolho KL (2014) Consensus guidelines of ECCO/ESPGHAN on the medical management of pediatric Crohn's disease.J Crohns Colitis 8(10):1179-1207

10. Amil-Dias J, Kolacek S, Turner D et al (2017) Surgical management of Crohn disease in children: guidelines from the paediatric IBD Porto group of ESPGHAN. J Pediatr Gastroenterol Nutr 64(5):818-835 
11. Grover Z, Muir R, Lewindon P (2014) Exclusive enteral nutrition induces early clinical, mucosal and transmuralremission in paediatric Crohn's disease. J Gastroenterol 49(4):638-645

12. Frivolt K, Schwerd T, Werkstetter KJ et al (2014) Repeated exclusive enteral nutrition in the treatment of paediatric crohn's disease: predictors of efficacy and outcome. Aliment Pharmacol Ther 39:1398-1407

13. Levine A, Wine E, Assa A et al (2019) Crohn's disease exclusion diet plus partial enteral nutrition induces sustained remission in a randomized controlled trial. Gastroenterology 157:440-450

14. Hyams JS, Dubinsky MC, Baldassano RN et al (2017) Infliximab is not associated with increased risk of malignancy or hemophagocytic lymphohistiocytosis in pediatric patients with inflammatory bowel disease. Gastroenterology 152(8):1901-1914

15. Jess T, Simonsen J, Jørgensen KT et al (2012) Decreasing risk of colorectal cancer in patients with inflammatory bowel disease over 30 years. Gastroenterology 143(2):375-381

16. Sceats LA, Dehghan MS, Rumer KK, Trickey A Morris AM, Kin C (2019) Surgery, stomas, and anxiety and depression in inflammatory bowel disease: a retrospective cohort analysis of privately insured patients. Colorectal Dis. https://doi.org/ 10.1111/codi.14905

17. Pachler FR, Brandsborg SB, Laurberg S (2017) Paradoxical impact of ileal pouch-anal anastomosis on male and female fertility in patients with ulcerative colitis. Dis Colon Rectum 60(6):603-607

18. Rajaratnam SG, Eglinton TW, Hider P, Fearnhead NS (2011) Impact of ileal pouch-anal anastomosis on female fertility: meta-analysis and systematic review. Int J Colorectal Dis 26(11):1365-1374

19. Loftus EV Jr, Guérin A, Yu AP, Wu EQ, Yang M, Chao J, Mulani PM (2011) Increased risks of developing anxiety and depression in young patients with Crohn's disease. Am J Gastroenterol 106(9):1670-1677

20. Mackner LM, Whitaker BN, Maddux MH et al (2020) Depression screening in pediatric inflammatory bowel disease clinics: recommendations and a toolkit for implementation. J Pediatr Gastroenterol Nutr 70(1):42-47

21. Brooks AJ, Rowse G, Ryder A, Peach EJ, Corfe BM, Lobo AJ (2016) Systematic review: psychological morbidity in young people with inflammatorybowel disease-risk factors and impacts. Aliment Pharmacol Ther 44(1):3-15

22. van den Brink G, Stapersma L, Vlug LE et al (2018) Clinical disease activity is associated with anxiety and depressive symptoms inadolescents and young adults with inflammatory bowel disease. Aliment Pharmacol Ther 48(3):358-369

23. Otley AR, Griffiths AM, Hale S (2006) Health-related quality of life in the first year after a diagnosis of pediatricinflammatory bowel disease. Inflamm Bowel Dis 12(8):684-691

24. Barnes EL, Kochar B, Long MD, Kappelman MD, Martin CF, Korzenik JR, Crockett SD (2017) Modifiable risk factors for hospital readmission among patients with inflammatorybowel disease in a nationwide database. Inflamm Bowel Dis 23(6):875-881

25. Stapersma L, van den Brink G, Szigethy EM, Escher JC, Utens EMWJ (2018) Systematic review with meta-analysis: anxiety and depression in children andadolescents with inflammatory bowel disease. Aliment Pharmacol Ther 48(5):496-506

26. Engelmann G, Erhard D, Petersen M et al (2015) Health-related quality of life in adolescents with inflammatory bowel diseasedepends on disease activity and psychiatric comorbidity. Child PsychiatryHum Dev 46(2):300-307

27. Szigethy EM, Youk AO, Benhayon D (2014) Depression subtypes in pediatric inflammatory bowel disease. J Pediatr Gastroenterol Nutr 58(5):574-581

28. Keethy D, Mrakotsky C, Szigethy E (2014) Pediatric inflammatory bowel disease and depres sion: treatment implications. Curr Opin Pediatr 26(5):561-567

29. LeLeiko NS, Lobato D, Hagin S et al (2013) Rates and predictors of oral medication adherence in pediatric patients with IBD. Inflamm Bowel Dis 19(4):832-839

30. Bennebroek Evertsz' F, Thijssens NA, Stokkers PC, Grootenhuis MA, Bockting CL, Nieuwkerk PT, Sprangers MA (2012) Do inflammatory bowel disease patients with anxiety and depressive symptomsreceive the care they need? J Crohns Colitis 6(1):68-76

31. Freckmann M, Seipp A, Laass MW et al (2018) School-related experience and performance with inflammatory bowel disease: results from a crosssectional survey in 675 children and their parents. BMJOpen Gastroenterol 5(1):e236 